\title{
EFFECTS OF ORGANOHALOGENATED XENOBIOTICS ON GUT MICROBIOTA, OXIDATIVE REDOX, AND REPRODUCTIVE FUNCTIONS IN PIGS - A REVIEW
}

\author{
LARTEY, K. A. ${ }^{1}-$ FAN, Y. ${ }^{1}-$ NIE, F.-H. ${ }^{2}-$ KANG, D.-J. ${ }^{1}-$ LIN, H.-Y. ${ }^{1}-$ NAMULA, Z. ${ }^{1}-$ CHEN, Z. \\ - WANG, H.-C. R. ${ }^{3}-$ GOONERATNE, R. ${ }^{4}-$ CHEN, J.-J. ${ }^{1 *}$ \\ ${ }^{I}$ Department of Veterinary Medicine, College of Coastal Agricultural Sciences, Guangdong \\ Ocean University, Zhanjiang, Guangdong 524088, China \\ ${ }^{2}$ Department of Food Safety, College of Food Science and Technology, Guangdong Ocean \\ University, Zhanjiang, Guangdong 524088, China \\ ${ }^{3}$ Department of Biomedical and Diagnostic Sciences, The College of Veterinary Medicine, \\ University of Tennessee, Knoxville TN 37996, USA \\ ${ }^{4}$ Department of Wine, Food and Molecular Biosciences, Faculty of Agriculture and Life \\ Sciences, Lincoln University, Lincoln 7647, New Zealand \\ *Corresponding author \\ e-mail:jjchen555@aliyun.com,chenjj@gdou.edu.cn
}

(Received $4^{\text {th }}$ Feb 2020; accepted $22^{\text {nd }}$ May 2020)

\begin{abstract}
Organohalogenated compounds contaminations in feed ingredients and feeds pose threats to the safety of food animals, and public health. Pigs are exposed through ingestions of feed contaminated with organohalogenated compounds. Microbes - organohalogens interactions in the gut cause changes in mean species diversities of bacteria, and induce gut dysbiosis. Along with metabolites from first-pass metabolisms, they affect proteins and molecular pathways that regulate ROS sensing, and induce oxidative stress. They also bind to estrogen receptors and mimic estrogen activities to impair reproductive endocrine functions. Nutritional interventions such as feed and feed ingredients substitutions, and harnessing non-conventional feed resources (NCFR) can offer sustainable alleviations. This will mitigate the risk of exposure to organohalogens whilst providing the needed nutrients to meet the animals' nutritional requirements. In addition, it will enhance the biological defense mechanisms in pigs. Phytonutrition can enhance biodegradation, and detoxification of recalcitrant organohalogenated xenobiotics. This provides a low cost, "green" strategy to alleviate adverse effects of organohalogenated xenobiotics in pigs. The low costs associated with makes this a viable remedy, especially for low income countries.
\end{abstract}

Keywords: feed ingredients, phytonutrition, dysbiosis, metabolism, xenoestrogen

\section{Introduction}

Pigs are the most widely consumed terrestrial food animals. Pig production is one of the fastest growing livestock enterprise. Pigs are efficient convertors of feeds to meats making pig production one of the most profitable livestock enterprise. Feeds and feed ingredients used in pig nutrition have been found to also contain xenobiotics such as organohalogens (Sapkota et al., 2007; Li et al., 2019). Organohalogenated compounds contaminations in feed ingredients and compound feeds pose threats to health, and performance of food animals, and meat safety and public health (Bernard et al., 2002; Barone et al., 2019; Das et al., 2019).

Organohalogens are ecotoxins produced as results of anthropogenic, biogenic, and geogenic activities (Xu et al., 2013). Persistence organic pollutants (POPs), pesticides, 
and pharmaceutical and personal care products (PPCPs) and dibutyl phthalate (DBPs) are produced from industrial, agricultural, and domestic activities (Bakhiyi et al., 2018). Polychlorinated biphenyls (PCBs) deposits in marine sediments gets transported (through microplastics) into oceans as micro pollutants (Gerdes et al., 2019). They get adsorbed by benthos, and biomagnify in adipose tissues of fish and other marine organisms at high trophic levels (Fernández-González et al., 2013; Jamieson et al., 2017; Li et al., 2019). Feed processing, and feed additives inclusions may also result in organohalogens contamination (Zijlstra and Beltranena, 2013). Environmental pollutions are the main sources organohalogenated compounds contaminations in food chains (Li et al., 2019). Oral route is the primary means of exposure in animals through the ingestion of organohalogenated compounds contaminated feeds (Sapkota et al., 2007; Li et al., 2019).

The tripartite linkage between livestock, humans, and the environment makes them prone to ecotoxins contaminations (Rabinowitz and Conti, 2013). In the setting of our time, organohalogenated xenobiotics have assume importance in food animal productions due to interdependence between livestock and the environment (Malisch, 2017). Humans and animals share health risk from environmental pollutions and zoonosis (Watanabe et al., 2010; Rabinowitz and Conti, 2013). There are increasing evidence of organohalogenated xenobiotics-induced degenerative diseases, and reproductive disorders in animals and humans (Barthold et al., 1999; Rabinowitz and Conti, 2013). Organohalogenated xenobiotic exposures in animals increase the risk of zoonosis (Barthold et al., 1999; Watanabe et al., 2010; Rabinowitz and Conti, 2013). Organohalogenated compounds have significant toxic effects even at low contaminations due to their ability to biomagnify, persist and bioaccumulate in food chains (El-Shahawi et al., 2010). Animal nutrition should therefore be critical component of comprehensive interdisciplinary preventive health strategies stipulated as in "One health" concept (Muthuvel et al., 2006). Nutritional interventions can modify the gut microbiome, and enhance the ability of (gut) bacteria to metabolize organohalogenated xenobiotics (Zhang et al., 2015b; Jin et al., 2017; Petriello et al., 2018). Utilizing plants bioactive compounds as immuno-nutritional supplement is an important nutritional intervention. The main therapeutic strategy is modulations of microbial compositions in the gut to enhance xenobiotics metabolisms (Petriello et al., 2014).

Homeostasis in the gut microbiota has been found to be critical in ensuring optimum immune, metabolic, and endocrine functions in pigs (Kim and Isaacson, 2015; Everaert et al., 2017; Patil et al., 2019). The gut serves as host to bacteria of different taxonomic diversities, referred to as microbiota (Mwaikono et al., 2018; Patil et al., 2019). The composition of bacteria taxa, and their metabolic functions defines the gut metagenome (Kim and Isaacson, 2015; Patil et al., 2019). First -pass (pre-systemic) metabolism of halogenated xenobiotics in the gut and liver produces toxic metabolites (Grimm et al., 2015). These metabolites are stored in hepatocytes, and endocrinocytes (Grimm et al., 2015).

Studies from our laboratory evaluated the adverse effects of PCBs extracted from the Zhanjiang marine offshore sediments on molecular pathways involved in gastrointestinal, metabolic, and development abnormalities in zebrafish (Liu et al., 2016; Nie et al., 2016; Yu et al., 2017). Mice have also been used as model animals to study the effect of organohalogenated xenobiotics on gut microbiota (Petriello et al., 2018; Chi et al., 2019). There is a homology in morphological, biochemical, and 
physiological characteristics between these model animals and some food animals (Hill et al., 2005; Chi et al., 2019). This review highlights potential effect of PCBs on underlying physiological mechanisms involved in gut health, cellular oxidative redox, and reproductive functions in pigs. Pigs are susceptible to PCB exposure, bioaccumulation, and biomagnification due to contaminations of their feeds, and bioaccumulations in their fatty tissues (Hoogenboom et al., 2004; Weber et al., 2018; Barone et al., 2019). The gut metagenome of pigs is important due to the influence on immune developments, and (reproductive) endocrine functions (Mach et al., 2015). It is also critical at post-weaning to prevent oxidative stress, a key pathophysiological disorder in pigs (Round and Mazmanian, 2009; Kim and Isaacson, 2015). Based on evidence from animal models and in vitro experiments, we discussed the potential effects of organohalogenated xenobiotics on gut abnormality, oxidative stress, and reproductive malfunctions in pigs. Nutritional, and husbandry interventions for "green", safe, and sustainable alleviations are also suggested in this review. This will also add to literature, as well as offer basis for future scientific research and interventions to alleviate the adverse effects of the organohalogenated compounds in pigs to enhance animal health, and public health.

\section{Organohalogenated compounds pollutions}

Organohalogenated compounds have acidic, alkaline, and thermal resistance $(\mathrm{Xu}$ et al., 2013). They were therefore used as coolants, and insulators in capacitors, flameretardants, plasticizers, and a host of industrial materials (Xu et al., 2013; Bakhiyi et al., 2018). Organochlorines pesticides were used to control insect pests to improve public health, and agricultural productivities (Xu et al., 2013; Bakhiyi et al., 2018). In addition to these anthropogenic sources, geogenic events such as volcanos, and wildfires also produce polychlorinated dibenzop-dioxins and furans (PCDD/F), referred to as dioxins. Biogenic activities such as the biodegradation and biotransformation of some inorganic chemicals in biosolids also yield toxic organohalogens. Antibiotics such as fluoroquinolones, enrofloxacin, and florfenicol used for therapeutic, and subtherapeutic uses, also contain organohalogens (Fernández-González et al., 2013; Zijlstra and Beltranena, 2013).

Polychlorinated biphenyls (PCBs) largely refers to any class of organohalogenated compound prepared by a reaction of chlorine with biphenyl. Typical mixtures of PCBs contain over 100 compounds which are colorless, viscous liquids. PCBs are long-lived organic compounds, owing to their resistance to biological, photolytic, and chemical degradations (Xu et al., 2013). They are hydrophobic, and lipophilic, making them bioaccumulate, and biomagnify in fatty tissues. They have a long-range transportability making them widely present in almost every geographical location and environment, including areas they were not utilized (Bakhiyi et al., 2018). The Stockholm convention listed PCBs, and dioxins among the dirty dozen hazardous chemicals in the world (Xu et al., 2013). The chemical structure of PCB is shown in Figure 1.

Following the Stockholm convention in 2001, PCBs and dioxins production and utilizations have been banned, however secondary emissions from sinks, and stockpile in old gadgets continues to cause pollutions (Xu et al., 2013; Spongberg and Witter, 2007). Significant levels have been detected in marine offshore sediment in industrialized countries in the northern and middle latitude (Nie et al., 2016; Yu et al., 2017). It is worth mentioning that, over the years studies on persistent organic pollutants 
(POPS) have been largely focused on industrialized (and developed) regions in the northern and middle latitudes, where they were largely produced and utilized (Spongberg and Witter, 2007). However, evidence from tropical developing countries in the southern latitude have revealed significant POPs contaminations in sediments, and water bodies in these areas (Spongberg and Witter, 2007; Gioia et al., 2014). Electronic waste dumping and their improper recycling in these regions may largely account for this (Spongberg and Witter, 2007; Hogarh et al., 2012; Bakhiyi et al., 2018). PCBs concentrations have been found to be increasing in warm tropical regions in the southern latitude owing to the high water temperatures and rate of air/gas exchange $(\mathrm{Fu}$ and $\mathrm{Wu}, 2006$; Spongberg and Witter, 2007). This may partly account for the presence of highly halogenated and toxic congeners in the tropical warms regions (Spongberg and Witter, 2007). The atmospheric total sum ( $\left.\sum 190 \mathrm{PCB}\right)$ concentration in Agbogbloshie (in Ghana), an improper e-waste recycling hub in tropical Africa, was found to be as high as $4.64 \mathrm{ng} / \mathrm{m}^{3}$ (Hogarh et al., 2012). Concentration in plumes in the area was about $11.10 \mathrm{ng} / \mathrm{m}^{3}$ (Amoyaw-Osei et al., 2011). They may be carried as effluent into water bodies, and biomagnify through marine food web resulting in pollutions of marine-sourced feed resource (El-Shahawi et al., 2010).

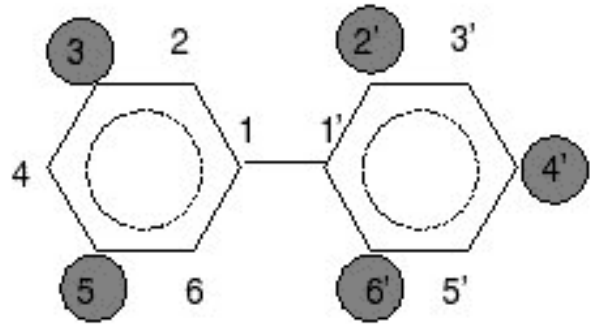

Figure 1. Chemical structure of PCBs

\section{Effect of organohalogenated xenobiotics on the gut microbiota of pigs}

Like most mammals, the pig's gut is the largest interface between their internal and external environments (Farhadi et al., 2003; Patil et al., 2019). It extends from the buccal cavity, passes through the intestines, and ends at the anal orifice. It contains the highest amount of bacteria of different taxonomies (Mach et al., 2015; Holmann et al., 2017). The term "gut metagenome usually includes the microbes and their metabolic interactions with the host (Patil et al., 2019). The gut bacterial ecology of pig is composed of 35\% Firmicutes, 21\% Bacteroidetes, 3\% Proteobacteria and 2\% Spirochetes (Kim and Isaacson, 2015; Patil et al., 2019). In the cecum and colon of pigs, firmicutes predominate at $75 \%$ or more, followed by proteobacteria at $13 \%$ (Kim and Isaacson, 2015; Patil et al., 2019). There is a mutually beneficial relationship between commensal bacteria and the host (Patil et al., 2019). The pigs' gut provide bacteria with nutrients, and energy for signal transductions (Kim and Isaacson, 2015). Short chain fatty acids (SCFAs) viz acetate, butyrate, and propionate are the ligands of guanine nucleotide-binding proteins (G-proteins), energy substrates for gluconeogenesis, and inhibitors of histone deacetylase (Layden et al., 2013). The probiotic (beneficial) bacteria such as Bifidobacteria longum and Lactobacillus casei biotransform primary bile acids (BA), produced in the liver, into secondary bile acids, to enable binding of the G-protein receptors to regulate intestinal barrier functions (Farhadi et al., 2003). This is important in resisting colonization of pathobionts, and preventing endotoxemia. The gut 
microbiota is sensitive to xenobiotics (Zhang et al., 2015b; Lefevera et al., 2016; Jin et al., 2017). The bacteria diversities exist in homeostatic state.

There are some similarities in the gut anatomy, physiologies, and biochemistry between pigs and laboratory animals such as mice, and zebrafish (Choi et al., 2010; Yu et al., 2017; Petriello et al., 2018). The observed effects of organohalogenated xenobiotics on their gut microbiota, and gut histology provide insights on the potential effects on gut abnormalities in pigs (Choi et al., 2010; Yu et al., 2017; Petriello et al., 2018). Ingested PCBs can therefore disrupt gut microbiota homeostasis, and reduce the mean species diversity as observed in mice (Choi et al., 2013; Petriello et al., 2018; Chi et al., 2019). Ingestion of $150 \mu \mathrm{mol} / \mathrm{kg}$ of 3 PCBs congenors (PCBs 153, 138, and 180) caused decreases in Proteobacteria species, and the overall abundance of bacteria in C57BL/6 mice (Petriello et al., 2018). They also caused a reduction in Firmicutes Bacteroidetes ratio in the cecum (Jin et al., 2017; Petriello et al., 2018) likely due to a surge in the relative abundance of Flavobacteria, and Clostridia (Petriello et al., 2018). 2,3,7,8- tetrachlorodibenzofuran caused an increase in the level of Flavobacteria in the gut of mice (Zhang et al., 2015b). Similarly, oral administration of a dioxin, 2,3,7,8tetrachlorodibenzo-p-dioxin at $24 \mu \mathrm{g} / \mathrm{kg}$ for 5 days caused an increase in the relative abundance of Butyrivibrio spp, and a decline in Oscillibacter spp level thereby resulting a decreased Firmicutes-Bacteroidetes ratio in cecal microbiota of mice (Zhang et al., $2015 \mathrm{~b}$ ). On the contrary, dioxin at a dose of $6 \mu \mathrm{g} / \mathrm{kg}$ biweekly for 26 weeks, increased Firmicutes/Bacteroidetes ratio, as results of increase in Lactobacillaceae and Desulfovibrionaceae levels and decrease in Prevotellaceae, without exacerbating streptozotocin-induced hyperglycemia in mice (Lefevera et al., 2016). It is therefore apparent that the degree of halogenation and toxicity have influence on the gut bacteria dynamics. Metabolic activities of gut bacteria are critical in biodegrading organohalogens xenobiotics, similar to soil bacteria degradations (Zhang et al., 2015a, b). Organohalide-respiring bacteria undergoes organohalide respiration to dehalogenate organohalogens (El-Shahawi et al., 2010; Zhang et al., 2015a, b; Jugder et al., 2016). The less toxic, less stable congeners further undergo aerobic and fermentative degradations to produce energy substrates such as carbon and phosphorous for the animal's biochemical and physiological processes (Zhang et al., 2015a, b). Genomes of Alistipes, Blautia, Eubacterium, Faecalibacterium, Roseburia and other core gut genera are reservoirs of (S)-2-haloacid dehalogenase genes (Shetty et al., 2017). They can chemically replace the halogen substitutes with hydrogen through a reduction reaction (Smidt and de Vos, 2004; Atashgahi et al., 2016, 2018). The reduction reaction biodegrades chemically stable (locked) organohalogens into less stable and less toxic congeners (Yim et al., 2008; Atashgahi et al., 2018). Clostridium spp (genus Desulfitobacterium) such as $C$. perfringens and $C$. beijerinckii may undergo metabolic reductive dehalogenation to dechlorinate hexachlorobiphenyl, and tetrachlorobiphenyl (toxic congeners) to pentachlorobiphenyl, and trichlorobiphenyl (Smidt and de Vos, 2004; Lefevera et al., 2006; Atashgahi et al., 2016; Jin et al., 2017). Mwaikono et al. (2018) characterized the fecal microbiota of dumpsite-scavenging pigs. Municipal dumpsites are potential sources of organohalogenated xenobiotics in the developing countries in Sub-Sahara Africa and other low-income countries (Watanabe et al., 2010). Using a high throughput Illumina MiSeq sequencing technology for 16S rRNA amplification, it was observed that the fecal microbiota of the scavenging pigs are characterized by the predominance of mobile genetic elements and pathogenic Proteobacteria (Watanabe et al., 2010). Increase in the relative abundance of the 
pathogenic phyla leads to disruption of gut microbiota homeostasis, changes in BA metabolism, and dysregulation of Farnesoid $\mathrm{X}$ receptor signaling pathways (Zhang et al., 2015b; Patil et al., 2019). This results in dysfunctional intestinal barriers, polydipsia and polyphagia, and liver toxicities (Lefevera et al., 2016; Petriello et al., 2018). Advances in the "omics" further support the corroboration that, a functional immune system is dependent on gut microbiome homeostasis (Round and Mazmanian, 2014; Lefevera et al., 2016; Chi et al., 2018). Suppression of antibodies production, and innate and adaptive immune responses have been linked to organohalogens-induced gut dysbiosis (Thomas and Hinsdill, 1978; Choi et al., 2010; Lefevera et al., 2016). Tolllike receptors (TLRs) are transmembrane glycoproteins involved in signal transduction to respond to active moiety of gram negative bacteria and endotoxins such as lipopolysaccharides (Round and Mazmanian, 2009; Lefevera et al., 2016). TLRs decrease in the jejunum and colon upon PCBs exposures in animals (Round and Mazmanian, 2009). Using Amarex-MT4 assay and turbidimetric immunoassay, Watanabe et al. (2010) suggested that the observed reduced plasma IgG and T4 levels in pigs scavenging on dumpsite was due to dioxin and related compounds pollutions in dumpsites. A metagenome analysis of fecal samples of pigs scavenging on dumpsites in Tanzania also showed expressions of functional pathways associated with biosynthesis of Staphylococcus aureus and other pathogenic infections of zoonotic potentials (Mwaikono et al., 2018). It is therefore apparent that, organohalogenated xenobiotic can cause gut dysbiosis, immunosuppression, and increase the risk of zoonotic diseases.

\section{Effects of organohalogenated xenobiotics on oxidative stress}

Gut dysbiosis from halogenated xenobiotics induce gut oxidative stress and systemic gut inflammations (Choi et al., 2010; Yu et al., 2017; Petriello et al., 2018). Gut oxidative phosphorylations, and cellular processes produce oxygen metabolites referred to as reactive oxygen species (ROS). These metabolites are by-products from partial reductions of oxygen (Ray et al., 2012; Buha et al., 2015). They include superoxide anion, hydroxyl radical, and hydrogen peroxide. At the oxidative interface, ROS may signal critical molecules for cell proliferation and survival (Ray et al., 2012). However, a disturbance in the oxidative redox causes oxidative imbalance, which induces oxidative stress (Ray et al., 2012; Buha et al., 2015).

Mechanism by which PCB ligands generates ROS is shown in Figure 2. Metabolism of organohalogenated xenobiotic produces four key hydroxylated metabolites viz polychlorobiphenylols (OH-PCBs), PCB-methylsulfones, PCB-catechols and PCBepoxides (James, 2001; James et al., 2008). They are potentially toxic due to their potential effects on hepatic physiologies (Grimm et al., 2015). Incomplete degradation and slow rate of biotransformation of highly halogenated and toxic congeners leads to the storage of these toxic metabolites in the hepatocytes (Selvakumar et al., 2013; Grimm et al., 2015). This affects proteins, and molecular pathways that regulate ROS sensing and metabolic processes necessary to maintain oxidative redox (Liu et al., 2014; Grimm et al., 2015). They activate Cytochrome P450 1A1 (CYP1A1) to facilitate detoxification (Barouki and Morel, 2001). Upregulations of CYP1A1 genes however generate induce toxic metabolites (Barouki and Morel, 2001). These metabolites bind to aryl hydrocarbon receptor (AhR) to suppress AhR expressions in the hepatocytes (Barouki and Morel, 2001; Dietrich et al., 2016; Nie et al., 2016; Nielsena et al., 2017). Suppression of AhR expressions to attenuate CYP1A1-inducing cytochrome P450s, 
generates excess hydroxyl radicals thereby resulting in oxidative imbalance (Selvakumar et al., 2013; Liu et al., 2014; Dietrich et al., 2016; Nielsena et al., 2017). In addition, the toxic metabolites decrease the synthesis of hepatic glutathione (Muthuvel et al., 2006), and also suppress activities of antioxidant enzymes such as SOD, GPx (Liu et al., 2014). They further increases stimulation of the pro-oxidants such as $\mathrm{Cu}$ (Liu et al., 2014; Hong et al., 2015; Nielsena et al., 2017). Metabolites from first-pass metabolism of organohalogens can therefore reduce antioxidant capacity and induce oxidative imbalance therefore causing oxidative stress in pigs. Proliferations of proinflammatory cytokines such as tumor necrosis factor alpha (TNF- $\alpha$ ) in the colon, ilea lesions, and intestinal inflammations have been observed in animal models upon exposure to PCBs and dioxins (Yu et al., 2017; Petriello et al., 2018). DL-PCBs caused a mild hydropic degeneration of epithelial cells in the intestine resulting in reduced intestinal folds in the gut of zebrafish larvae (Yu et al., 2017). Gut oxidative damage as result of PCBs exposure can damage, rupture or shed the intestinal villus in pigs (Choi et al., 2010; Brugman, 2016). Dioxin related compounds seem to have a potential to induce CYP1A1, and disrupt Peroxisome Proliferator-Activated Receptor (PPAR) signaling pathways in pigs (Watanabe et al., 2010; Liu et al., 2014). PPAR is critical for inflammatory response and hence disruption of the PPAR signaling pathway can cause inflammatory diseases in pigs. Oxidative stress in pigs has been identified as an underlying pathogenicity of several pathophysiological disorders such as inflammatory diseases, and heat stress in pigs (Lee et al., 2016).

\section{Effect of PCBs on reproductive functions}

The productivity, and profitability of pigs is largely as results of their high reproductive performance. Reproductive physiologies are regulated by folliculogenesis and steroidogenesis, which are driven by reproductive hormones (Pocar et al., 2011). Reproductive toxicants affect folliculogenesis and steroidogenesis, which impair reproductive functions at pre-, peri-, and post-natal stages (Pocar et al., 2011; Brevini et al., 2015).

Organohalogenated compounds share similar chemical properties with estrogens, and can bind to the estrogen receptors (Diamanti-Kandarakis et al., 2009; Nie et al., 2016; Sheng et al., 2019). They mimic estrogen activities, and this prevents estrogens from functioning properly (Brevini et al., 2015; Nie et al., 2016; Sheng et al., 2019). Findings from studies in our laboratory showed that, PCBs can induce EROD expression in zebrafish as results of Arh agonist mechanisms (Liu et al., 2016; Nie et al., 2016). Effects of the xenoestrogenic compounds on reproductive physiologies in vivo, and in-vitro provide insight into their potential adverse effects on reproductive functions in pigs (Miller et al., 2004; Hill et al., 2005). Exposure to pigs can therefore induce adverse reproductive malfunctions, fetal malformation, and development toxicities (Miller et al., 2004; Pocar et al., 2011). PCB 28, PCB 105, and aroclor 1221 affected the activities of P450 arom, and inhibited modulation of Follicle Stimulating Hormone-induced aromatase activity in vitro (Woodhouse and Cooke, 2004). Very low concentrations (0.001-0.01 microg/ml) of Acroclor-1254 decreased oocyte maturation and development of bastocytes in an in vitro cumulus-oocytes complex (Campagna et al., 2008). OH-PCBs from plasma of sows treated with PCB, at $42 \mathrm{mg} / \mathrm{l}$ also induced embryotoxicities in vitro (Campagna et al., 2008). Blastomeres per expanded blastocyst were reduced at dose dependent manner (Campagna et al., 2008). 
Results from these studies therefore suggest that, organohalogenated xenobiotics can delay puberty, shorten estrous, induce abortions, and still birth, and reduce litter size in sows (Woodhouse and Cooke, 2004; Brevini et al., 2005; Campagna et al., 2008). Intergeneration toxicities lasting into F3 generations are possible (Pocar et al., 2011). Exposure to Aroclor 1221 at low-levels $(0.01-0.1 \mu \mathrm{g} / \mathrm{ml})$ during the teratogenic sensitive period induced dysregulation and complex adverse effects on the physiology, fertility, and fecundity, and sex ratio of F1 and F2 two offspring in Sprague-Dawley rats (Steinberg et al., 2008). Abnormalities in testes weight, sperm count, sperm viability, seminiferous tubule diameter (in males), and adrenocorticosteroid, estrus period, ovary weight, follicular atresia, and oocyte developmental (in female) were observed in offspring when dams were exposed to PCBs (Miller et al., 2004; Pocar et al., 2011). These could be due to impaired growth and development at the embryonic stage (Père and Etienne, 2000; Pocar et al., 2011). Cryptorchidism has been reported in F1 pigs upon maternal exposure to toxic 2,3,7,8-TCDD (most toxic dioxin congener) during the utero and lactation stages (Barthold et al., 1999). Comparing the concentrations of DRCs between dumpsite scavenging pigs and their neonates, Watanabe et al. (2010) detected higher concentrations of higher molecular weight congeners in piglets, DRCs seem to have higher bioaccumulation in breastmilk than in serum. The effect of organohalogenated compounds on reproductive and development toxicities is shown in Figure 3.

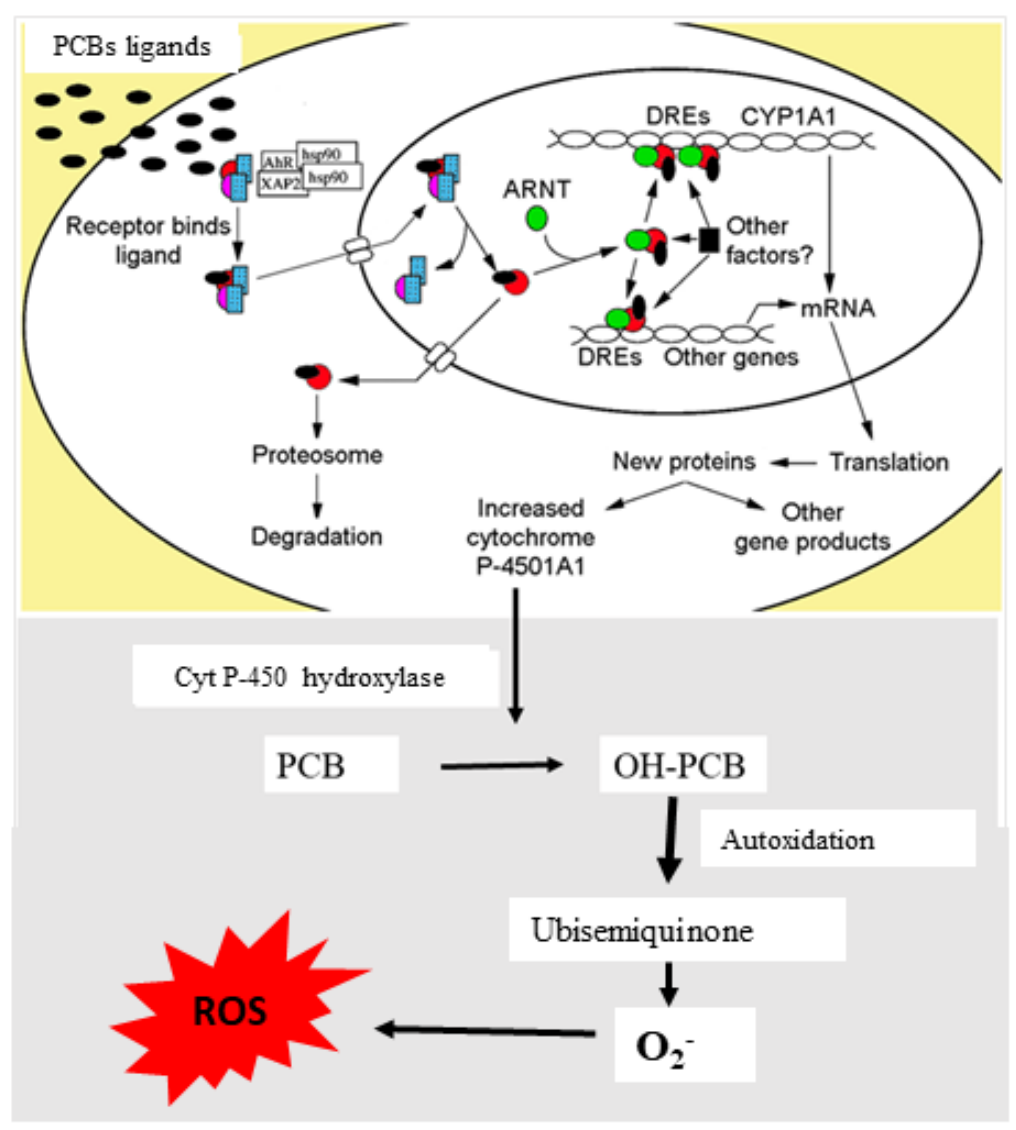

Figure 2. ROS generation mechanism induced by organohalogenated xenobiotics. ARNT: aryl hydrocarbon receptor nuclear translocator; XAP2: aryl hydrocarbon receptor interacting protein; hsp: heat shock protein; DRE: dioxin response element 


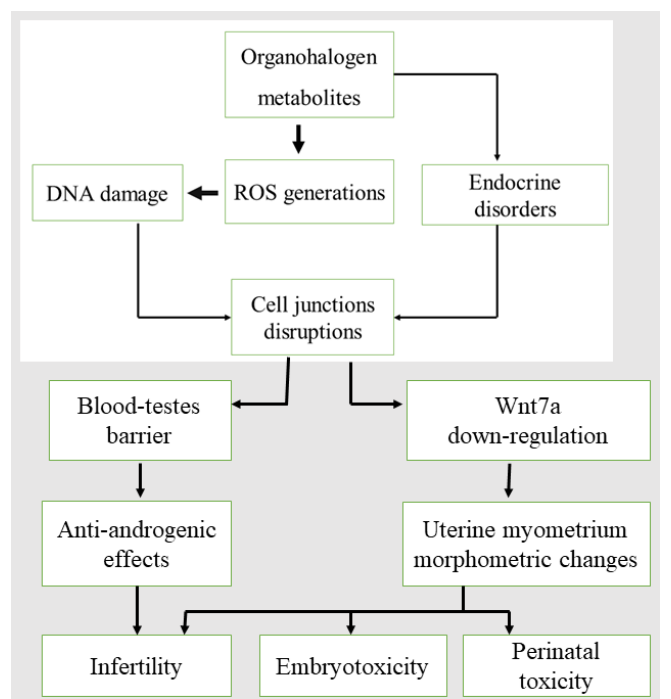

Figure 3. Schematic diagram on reproductive malfunctions induce by organohalogenated xenobiotics

\section{Exposure of pigs to organohalogenated xenobiotics}

Xenobiotics in animals refer to any synthetic or naturally occurring chemical substances present in the animal that are not naturally synthesized by the animal, and are hence considered foreign agents to the animal's body (Atashgahi et al., 2018). Oral intake is the commonest and primary means of exposure in farm animals (see Fig. 4). Inhalation from polluted air, and ocular, and dermal contacts may also occur. PCBs may be absorbed as micropollutants $(\mathrm{ng} / \mathrm{L}-\mu \mathrm{g} / \mathrm{L})$ or at higher range $(\mu \mathrm{g} / \mathrm{L}-\mathrm{mg} / \mathrm{L})$. It occurs as means of ingestion of organohalogenated contaminated feeds (Hoogenboom et al., 2004). Total equivalent quantity of DLPCBs (TEQ DL-PCBs) detected in feed samples from 16 feed ingredients was found to be $1.3 \mathrm{ng} / \mathrm{kg}$ (Fernández-González et al., 2013). This is slightly lower than the maximum level of $1.5 \mathrm{ng} / \mathrm{kg}$ TEQDL-PCBs for feeds as specified by the European Union as shown in Table 1 (Fernández-González et al., 2013). TEQs PCDDs, PCDFs and Co-PCBs of pig feed samples in Japan was also found to be in a range of 0.13 to $0.32 \mathrm{pg}$ g-1 lipid wt (Guruge et al., 2005). Animal fat (ANFA) feeds contain the highest total of PCBs (Weber et al., 2018), about 5 times higher than vegetable oil feeds (Fernández-González et al., 2013). This is largely due to the bioaccumulation of organohalogenated compounds in marine animal feed resources. Fish meals, fish oil, and shell powder have higher amounts of organohalogenated compounds compared to plant (vegetable) sources as shown in Figure 5 (Hoogenboom, 2004). Figure 5 shows PCB contaminations in sampled animal feeds, and feed ingredients.

Organohalogenated xenobiotics can also be transferred from lactating dams to suckling piglets at breastfeeding. The high levels of fat in piglets makes them susceptible to organohalogenated compounds bioaccumulation, and biomagnification. Biomagnification factors of 14 , and 15 were observed for 2,3,7,8-TeCDF, and 1,2,3,7,8PeCDD and 1,2,3,4,7,8- HxCDF respectively in fat of pigs (Traag et al., 2001). The biomagnification factor represents the ratio of lipid weight animal fat to mixed feed concentration. Exposure of pigs to organohalogens results in bioaccumulation in the back fat of pigs (Hoogenboom et al., 2004). Starter pigs exposed to a 10-fold contaminated feed from the recycled fat pollution (in Europe) resulted in $123 \mathrm{ng}$ 
TEQ/kg PCBs in back fat (Hoogenboom et al., 2004). A survey on meats in France found PCBs levels in pig meats to be higher (41.8-77.7 $\mathrm{ng} \mathrm{g}^{-1}$ live weight) than the EU maximum permissible levels (Barone et al., 2019). Therefore, incomplete metabolism of organohalogens xenobiotics can affect pig meat safety, in addition to the physiological and immune disorders.

\section{Sustainable alleviation strategies}

Dietary inclusion of fish meal, fish oils, and shell powder are necessary to meet pigs' demands for vitamins, digestible proteins, micronutrients, and essential fatty acids to ensure proper physiological, and immune developments in pigs (Kim and Easter, 2001; Fernández-González et al., 2013). Fish meal, and fish oil particularly contains eicosapentaenoic acid and docosahexaenoic acid, omega-3 long-chain polyunsaturated fatty acids (Kouba and Mourot, 2011; Hong et al., 2015; Saini and Keum, 2018). Animal feeds serve as a major linkage between animals and environment in the transfer of ecotoxins, through ingestion of contaminated feeds (Mizukawa et al., 2015). Safer animal nutrition and preventive health therefore needs to be important management strategies in alleviating effects of organohalogenated xenobiotics in pigs (Muthuvel et al., 2006; James et al., 2008; Zingsstag et al., 2011; Petriello et al., 2016). Improved animal husbandry and preventive animal health strategies aimed at mitigating animals' exposures to organohalogenated compounds, and enhancing their biology defenses have therefore become necessary (Muthuvel et al., 2006; James et al., 2008; Zinsstag et al., 2011; Dey et al., 2019).

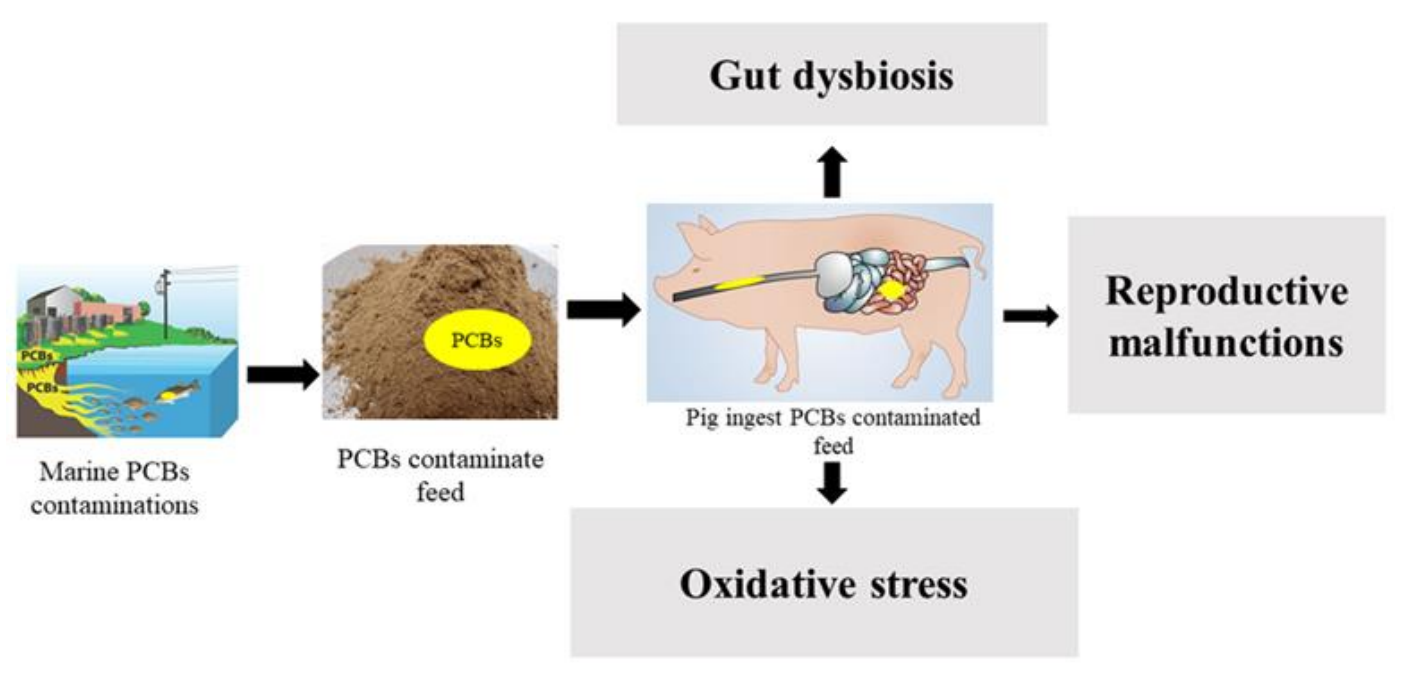

Figure 4. Exposure of pigs to PCB contaminated fish meal

Safety evaluation of animal feed and feed ingredients have gained important recognition in animal nutrition, and public health since feed and feed ingredient are critical components of the supply chains of (animal-derived) foods (PiskorskaPliszczynska et al., 2019). Above the threshold levels in feeds, toxicants pose adverse effects in food animals. Ensuring that PCB levels in animal feeds are below the threshold levels is an important feed safety evaluation protocol. Maximum levels, and action levels of dioxins and DLPCBs in feed ingredients have been studied using GC- 
MS/MS based analytical tools. Action levels in feeds and feed ingredients are lower than the maximum values, and serve as a safer tool for early warning and detection of higher than desirable levels of PCBs in animal feed and feed ingredients (Malisch, 2017). At levels exceeding the maximum levels, feed ingredients and animal feed are prevented from entering the food chains in Europe. At levels closer or above the action level in feed (ingredients), investigations and tracing of contaminants are conducted to identify the sources of contaminations. The maximum levels and action levels of some common feed ingredient used for diets of pigs are shown in Table 1. This is based on the European Union maximum levels, and action thresholds of PCBs for feed ingredients laid down in Directive 2002/32/EC and amended by Commission Regulation (EU) 2019/1869 (Piskorska-Pliszczynska et al., 2019).
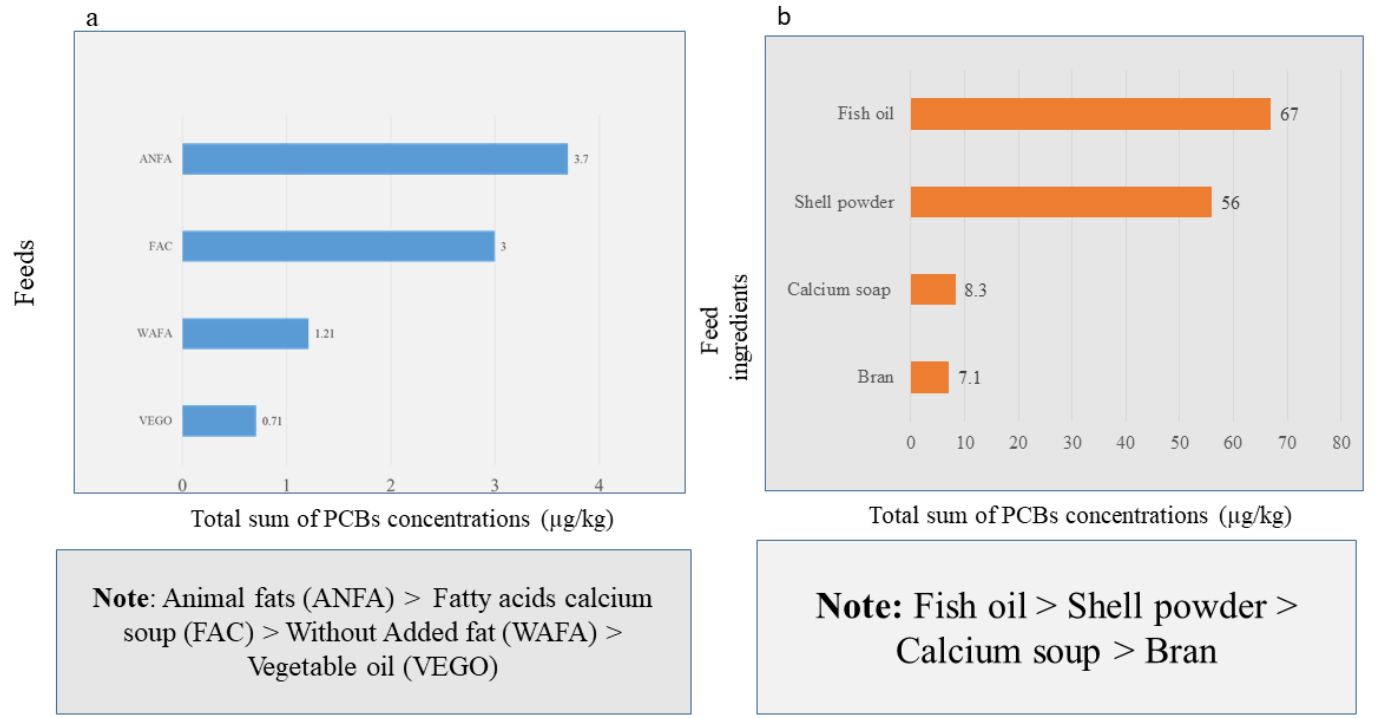

Figure 5. Total sum of PCBs concentrations ( $\Sigma$ PCBs) is feeds $(a)$ and feed ingredients $(b)$ (Fernández-González et al., 2013)

Table 1. Maximum levels and action level of dioxin and DLPCBs in some feed ingredients (at moisture content of 12\%)

\begin{tabular}{c|c|c|c|c}
\hline \multirow{2}{*}{ Feed and feed ingredients } & \multicolumn{2}{|c|}{ Maximum levels } & \multicolumn{2}{c}{ Action level } \\
\cline { 2 - 5 } & Dioxins (ng/kg) & $\begin{array}{c}\boldsymbol{\Sigma}_{\text {Dioxin }+ \text { DLPCBs }} \\
(\mathbf{n g} / \mathbf{k g})\end{array}$ & Dioxins (ng/kg) & $\boldsymbol{\Sigma}_{\text {DLPCBs }}(\mathbf{n g} / \mathbf{k g})$ \\
\hline Cereals & 0.5 & 0.35 & 0.75 & 1.25 \\
Soybean oils & 0.5 & 0.5 & 0.75 & 1.5 \\
Soybean meal & 0.5 & 0.5 & 0.75 & 1.5 \\
Calcium soaps & 0.5 & 0.35 & 0.75 & 1 \\
Egg shell meal & 0.5 & 0.35 & 0.75 & 1.25 \\
Fish oil & 4 & 11 & 5 & 20 \\
Fish meal & 1.25 & 5 & 1.75 & 9 \\
Oyster shell powder & 1.25 & 2 & 1.75 & 4 \\
Salts & 0.5 & 0.5 & 1 & 1.5 \\
Vitamin premix & 0.5 & 0.35 & 0.75 & 1.5 \\
Compound feed & 0.5 & 0.5 & & \\
\hline
\end{tabular}


Manipulating dietary compositions to reduce the total PCB levels in feed can be an important safety protocol to mitigate exposures to pigs (Sapkota et al., 2007; Petriello et al., 2016). Substituting feed ingredients with high contaminations with less contaminated feed ingredients provides an important nutritional intervention to reduce organohalogenated compounds exposure in food animals. Replacing shell powder which are sourced from crustaceans, with egg shell powder sourced from poultry (land) can reduce organohalogenated compounds levels in animal feed since marine-origin feed bioresources have relative high contaminations (Sapkota et al., 2007; James et al., 2008; Fernández-González et al., 2013; Petriello et al., 2014; Li et al., 2019). Animalderived feed ingredients can be replaced with plant-based ones. Replacing fishmeal, and fish oil with vegetable proteins, and vegetable oils can reduce the concentration of PCBs in animal feed. In addition, husbandry systems that expose food animals to the environment increase their exposure to ecotoxins (Piskorska-Pliszczynska et al., 2019). Unlike Europe and other developed countries where pig productions are characterized by specialized industrial production systems, productions in Sub-Sahara Africa and other low-income countries are a kind of traditional subsistence small-scale production characterized by outdoor management systems (Watanabe et al., 2010). This increases the risk of the food animal to organohalogenated compounds exposures due to the relative higher contact with the environment. Unlike confined pigs in intensive management systems, scavenging pigs in outdoor management systems burrow and wallow on dumpsites and sewerage sledges contaminated with PCBs (Mwaikono et al., 2018). Therefore, subsistence indoor pig management systems are necessary to mitigate exposure of the food animal to the environment and ecotoxins in low income countries. Simple housings systems using locally available materials have become necessary to mitigate exposures in pigs.

In addition, utilizing agro-food by-products such as groundnut cake and palm kernel cake, crop residues such as legume haulms and culled vegetables, and leaf meals from nutrient-rich herbal plants such as Alfalfa (Medicago sativa) and Siam weed (Chromolaena odorata) can also enhance feed safety. Such non-convectional feeds are safe, cheap, and environmental-friendly and hence suitable feed for pig nutrition in tropical low-income countries. Proximate analysis of such feeds have shown that they contain significant amount macro, micro-, and phyto-nutrients (Apori et al., 2000; Achilonu et al., 2018).

Phytonutrition can further enhance immunity, and biological defense systems of pigs against PCBs (Muthuvel et al., 2006; James et al., 2008; Gupta et al., 2014; Petriello et al., 2016; Dey et al., 2019). Extracts and powders from plants contain significant bioactive compounds (polyphenols), and are potential immuno-nutritional supplements against halogenated xenobiotics for sustainable pig productions (Petriello et al., 2014). They can stimulate eubiosis, xenobiotic metabolism, and steroidogenesis and folliculogenesis to enhance gut microbiota, oxidative redox, and reproductive functions in pigs (Gupta and Prakash, 2014; Dey et al., 2019). 3,3'-Di-indolylmethane, a glucosinolate from cruciferous vegetables (such as cabbage), stimulates hydroxylation, and detoxification through glucuronidation of UDP glucuronosyltransferase 1 family (UGT1A1) (James et al., 2008). An integration of such herbal feed additives, and leaf meals in pig nutrition can provide low cost, and efficient "green" technology to alleviate effects of organohalogenated xenobiotics in pigs (James et al., 2008; Gupta and Prakash, 2014; Petriello et al., 2014; Achilonu et al., 2018). 


\section{Conclusion}

PCBs and dioxins contaminations in feeds and feed ingredients expose pigs to organohalogenated xenobiotics. Pigs have a high magnification factor for organohalogenated xenobiotics. Enterohepatic circulations of organohalogenated xenobiotics induce gastrointestinal dysbiosis, and cellular oxidative stress. Metabolites of the endocrine disruptions compounds further induce reproductive and development abnormalities in pigs. Alleviating the adverse effects of organohalogenated xenobiotics in pigs requires nutritional interventions. Non-convectional feed resources can be harnessed to reduce pigs' exposure to organohalogenated xenobiotics whilst meeting their nutritional needs. Plants bioactive compounds also offer reliable immunonutritional supplements to enhance xenobiotics metabolisms, and immune-potentiation, and endocrine functions.

Acknowledgements. Supported by Marine Economy Development Project of Guangdong Province for High Quality Economic Development (GDOE2019A52).

\section{REFERENCES}

[1] Achilonu, M., Shale, K., Arthur, G., Naidoo, K., Mbatha, M. (2018): Phytochemical benefits of agroresidues as alternative nutritive dietary resource for pig and poultry farming. - Journal of Chemistry Article ID 1035071.

[2] Amoyaw-Osei, Y., Agyekum, O. O., Pwamang, J. A., Mueller, E., Fasko, R., Schluep, M. (2011): Ghana E-Waste Country Assessment. - SBC e-Waste Africa Project.

[3] Apori, S. O., Long, R. J., Castro, F. B., Ørskov, E. R. (2000): Chemical composition and nutritive value of leaves and stems of tropical weed Chromolaena odorata. - Grass and Forage Science 55: 77-81.

[4] Atashgahi, S., Lu, Y., Smidt, H. (2016): Overview of Known Organohalide-Respiring Bacteria - Phylogenetic Diversity and Environmental Distribution. - In: Adrian, L., Löffler, F. (eds.) Organohalide-Respiring Bacteria. Springer-Verlag, Berlin, pp. 63-105.

[5] Atashgahi, S., Liebensteiner, M. G., Janssen, D. B., Smidt, H., Stams, A. J. M., Sipkema D (2018): Microbial synthesis and transformation of inorganic and organic chlorine compounds. - Frontiers of Microbiology 9: 3079.

[6] Bakhiyi, B., Gravel, S., Ceballos, D., Flynn, M. A., Zayed, J. (2018): Has the question of e-waste opened a Pandora's box? An overview of unpredictable issues and challenges. Environment International 110: 173-192.

[7] Barone, G., Storellia, A., Nicoletta, C., Quaglia, N. C., Dambrosio, A., Garofalo, R., Chiumarulo, R. M. M. (2019): Dioxin and PCB residues in meats from Italy: consumer dietary exposure. - Food and Chemical Toxicology 133: 110717.

[8] Barouki, R., Morel, Y. (2001): Repression of cytochrome P450 1A1 gene expression by oxidative stress: mechanisms and biological implications. - Biochemical Pharmacology 61: 511-516.

[9] Barthold, J. S., Kryger, J. V., Derusha, A. M., Duel, B. P., Jednak, R., Skafar, D. F. (1999): Effects of an environmental endocrine disruptor on fetal development, estrogen receptor (alpha) and epidermal growth factor receptor expression in the porcine male genital tract. - The Journal of Urology 162: 864-871.

[10] Bernard, A., Broeckaert, F., De Poorter, G., De Cock, A., Hermans, C., Saegerman, C., Houins, G. (2002): The Belgian PCB/dioxin incident: analysis of the food chain contamination and health risk evaluation. - Environmental Research 88: 1-18. 
[11] Brevini, T., Cillo, F., Antonini, S., Gandolfi, F. (2005): Effects of endocrine disrupters on the oocytes and embryos of farm animals. - Reproduction in Domestic Animals 40: 291299.

[12] Brugman, S. (2016): The zebrafish as a model to study intestinal inflammation. Developmental \& Comparative Immunology 64: 82-92.

[13] Buha, A., Antonijević, B., Milovanović, V., Janković, S., Bulat, Z., Matović, V. (2015): Polychlorinated biphenyls as oxidative stress inducers in liver of sub acutely exposed rats: implication for dose-dependence toxicity and benchmark dose concept. Environmental Research 136: 309-317.

[14] Campagna, C., Ayotte, P., Sirard, M., Bailey, J. (2008): An environmentally relevant mixture of organochlorines, their metabolites and effects on preimplantation development of porcine embryos. - Reproductive Toxicology 25: 361-366.

[15] Chi, Y., Lin, Y., Lu, Y., Huang, Q., Ye, G., Dong, S. (2018): Gut microbiota dysbiosis correlates with a low-dose PCB126-induced dyslipidemia and non-alcoholic fatty liver disease. - Science of the Total Environment 653: 274-282.

[16] Choi, Y. J., Seelbach, M. J., Pu, H., Eum, S. Y., Chen, L., Zhang, B., Hennig, B., Toborek, M. (2010): Polychlorinated Biphenyls disrupt intestinal integrity via NADPH Oxidase-induced alterations of tight junction protein expression. - Environmental Health Perspectives 118: 976-981.

[17] Das, A. K., Nanda, P. K., Das, A., Biswas, S. (2019): Hazards and safety issues of meat and meat products. - Food Safety and Human Health 6: 145-168.

[18] Dey, P. (2019): Gut microbiota in phytopharmacology: a comprehensive overview of concepts, reciprocal interactions, biotransformations and mode of actions. Pharmacological Research 147: 104367.

[19] Diamanti-Kandarakis, E., Bourguignon, J. P., Giudice, L. C., Hauser, R., Prins, G. S., Soto, A. M., Zoeller, R. T., Gore, A. C. (2009): Endocrine-disrupting chemicals: an endocrine society scientific statement. - Endocrine Reviews 30: 293-342.

[20] El-Shahawi, M. S., Hamza, A., Bashammakh, A. S., Al-Saggafa, W. T. (2010): An overview on the accumulation, distribution, transformations, toxicity and analytical methods for the monitoring of persistent organic pollutants. - Talanta 80: 1587-1597.

[21] Everaert, N., Van Cruchten, S., Weström, B., Bailey, M., Van Ginneken., C., Thymann, T., Pieper, R. (2017): A review on early gut maturation and colonization in pigs, including biological and dietary factors affecting gut homeostasis. - Animal Feed Science and Technology 233: 89-103.

[22] Farhadi, A., Banan, A., Fields, J., Keshavarzian, A. (2003): Intestinal barrier: an interface between health and disease. - Journal of Gastroenterology and Hepatology 18: 479-497.

[23] Fernández-González, R., Yebra-Pimentel, I., Martínez-Carballo, E., Regueiro, J., SimalGándara, J (2013): Inputs of polychlorinated biphenyl residues in animal feeds. - Food Chemistry 140: 1-15.

[24] Gerdes, Z., Ogonowski, M., Nybom, I., Ek, C., Adolfsson-Erici, M., Barth, A., Gorokhova, E. (2019): Microplastic-mediated transport of PCBs? A depuration study with Daphnia magna. - PLoS ONE 14(2): e0205378.

[25] Gioia, R., Akindele, A. J., Adebusoye, S. A., Asante, K. A., Tanabe, S., Buekens, A., Sasco, A. J (2014): Polychlorinated biphenyls (PCBs) in Africa: a review of environmental levels. - Environmental Science and Pollution Research 21: 6278-6289.

[26] Grimm, F. A., Hu, D., Kania-Korwel, I., Lehmler, H. J., Ludewig, G., Hornbuckle, K. C., Duffel, M. W., Bergman, A., Robertson, L. W. (2015): Metabolism and metabolites of polychlorinated biphenyls (PCBs). - Critical Reviews in Toxicology 45: 245-272.

[27] Gupta, C., Prakash, D. (2014): Phytonutrients as therapeutic agents. - Journal of Complementary and Integrative Medicine 11: 151-169.

[28] Guruge, K. S., Seike, N., Yamanaka, N., Miyazakia, S. (2005): Polychlorinated dibenzop-dioxins, -dibenzofurans, and biphenyls in domestic animal food stuff and their fat. Chemosphere 58: 883-889. 
[29] Hill, A. J., Teraoka, H., Heideman, W., Peterson, R. E. (2005): Zebrafish as a Model Vertebrate for Investigating Chemical Toxicity. - Toxicological Sciences 86: 6-19.

[30] Hogarh, J. N., Seike, N., Kobara, Y., Masunaga, S. (2012): Atmospheric polychlorinated naphthalenes in Ghana. - Environmental Science \& Technology 46: 2600-2606.

[31] Holman, D. B., Brunelle, B. W., Trachsel, J., Allen, H. K. (2017): Meta-analysis to define a core microbiota in the swine gut. - mSystems 2: e00004-17.

[32] Hong, M. Y., Lumibao, J., Mistry, P., Saleh, R., Hoh, E. (2015): Fish oil contaminated with persistent organic pollutants reduces antioxidant capacity and induces oxidative stress without affecting its capacity to lower lipid concentrations and systemic inflammation in rats. - Journal of Nutrition 145: 939-944.

[33] Hoogenboom, A. P., Kan, C., Bovee, T. F. H., van der Weg, G., Onstenk, C., Traag, W. A. (2004): Residues of dioxins and PCBs in fat of growing pigs and broilers fed contaminated feed. - Chemosphere 57: 35-42.

[34] James, M. O. (2001): Polychlorinated Biphenyls: Metabolism and Metabolites. - In: Robertson, L. W., Hansen, L. G. (eds.) PCBs - Recent Advances in Environmental Toxicology and Health Effects. University of Kentucky Press, Lexington, pp. 35-46.

[35] James, M. O., Sacco, J. C., Faux, L. R. (2008): Effects of food natural products on the biotransformation of PCBs. - Environmental Toxicology and Pharmacology 25: 211-217.

[36] Jamieson, A. J., Malkocs, T., Piertney, S. B., Fujii, T., Zhang, Z. (2017): Bioaccumulation of persistent organic pollutants in the deepest ocean fauna. - Nature Ecology \& Evolution 1: 0051.

[37] Jin, Y., Wu, S., Zeng, Z., Fu, Z. (2017): Effects of environmental pollutants on gut microbiota. - Environmental Pollution 222: 1-9.

[38] Jugder, B. E., Ertan, H., Susanne, Bohl, S., Lee, M., Marquis, C. P., Manefield, M. (2016): Organohalide respiring bacteria and reductive dehalogenases: key tools in organohalide bioremediation. - Frontiers in Microbiology, 7: 249.

[39] Kim, H. B., Isaacson, R. E. (2015): The pig gut microbial diversity: understanding the pig gut microbial ecology through the next generation high throughput sequencing. Veterinary Microbiology 177: 242-251.

[40] Kouba, M., Mourot, J. (2011): A review of nutritional effects on fat composition of animal products with special emphasis on n-3 polyunsaturated fatty acids. - Biochimie 93: 13-17.

[41] Layden, B. T., Angueira, A. R., Brodsky, M., Durai, V., Lowe, W. L. (2013): Short chain fatty acids and their receptors: new metabolic targets. - Translational Research 161: 131140.

[42] Lee, I. K., Kye, C. Y., Kim, G., Kim, H. W., Gu, M. J., Umboh, J., Maaruf, K., Kim, S. W., Yun, C. H. (2016): Stress, nutrition, and intestinal immune responses in pigs - A Review. - Asian Australasian Journal of Animal Sciences 29: 1075-1082.

[43] Lefevera, D. E., Xu, J., Chen, Y., Huang, G., Tamas, N., Guo, T. L. (2016): TCDD modulation of gut microbiome correlated with liver and immune toxicity in streptozotocin (STZ)-induced hyperglycemic mice. - Toxicology and Applied Pharmacology 304: 48-58.

[44] Li, X., Dong, S., Wang, P., Xiao, O., Su, X., Fu, J. (2019): Polychlorinated biphenyls are still alarming persistent organic pollutants in marine-origin animal feed (fishmeal). Chemosphere 233: 355-362.

[45] Liu, H., Nie, F., Lin, H., Ma, Y., Ju, X., Chen, J., Gooneratne, R. (2014): Developmental toxicity, EROD, and CYP1A mRNA expression in zebrafish embryos exposed to DioxinLike PCB126. - Environmental Toxicology 31: 295-303.

[46] Mach, N., Berri, M., Estellé, J., Levenez, F., Lemonnier, G., Denis, C., Leplat, J. J., Chevaleyre, C., Billon, Y., Doré, J., Rogel-Gaillard, C., Lepage, P. (2015): Early-life establishment of the swine gut microbiome and impact on host phenotype. Environmental Microbiology Reports 7: 554-56. 
[47] Miller, K. P., Borgeest, C., Greenfeld, C., Tomic, D., Flaws, J. A. (2004): In utero effects of chemicals on reproductive tissues in females. - Toxicology and Applied Pharmacology 198: 111-131.

[48] Mizukawa, H., Nomiyama, K., Kunisue, T., Watanabe, M. X., Subramanian, A., Iwata, H., Ishizuka, M., Tanabe, S. (2015): Organohalogens and their hydroxylated metabolites in the blood of pigs from an open waste dumping site in south India: association with hepatic cytochrome P450. - Environmental Research 138: 255-263.

[49] Muthuvel, R., Venkataraman, P., Krishnamoorthy, G., Gunadharini, D. N., Kanagaraj, P., Stanley, A. J., Srinivasan, N., Balasubramanian, K., Aruldhas, M. M., Arunakaran, J. (2006): Antioxidant effect of ascorbic acid on PCB (Aroclor 1254) induced oxidative stress in hypothalamus of albino rats. - Clinica Chimica Acta 365: 297-303.

[50] Nie, F., Cai, J., Wang, X., Lin, H., Gooneratne, R., Hay, A., Ma, Y., Ju, X., Zheng, J., Chen, J. J (2016): EROD activity and CYP1A mRNA expression in zebrafish embryo exposed to marine sediment DLPCBs extract. - Asian Journal of Ecotoxicology 11: 364368 (English abstract).

[51] Nielsena, S. D., Bauhausa, Y., Zamaratskaia, G., Junqueira, M. A., Blaabjerg, K., PetratMelin, B., Young, J. F., Rasmussen, M. K. (2017): Constitutive expression and activity of cytochrome P450 in conventional pigs. - Research in Veterinary Science 111: 75-80.

[52] Patil, Y., Gooneratne, R., Ju, X. H. (2019): Interactions between host and gut microbiota in domestic pigs: a review. - Gut Microbe 2019: 1-25.

[53] Père, M. C., Etienne, M. (2000): Uterine blood flow in sows: effects of pregnancy stage and litter size. - Reproduction Nutrition Development 40: 369-382.

[54] Petriello, M. C., Bradley, J., Newsome, B. J., Dziubla, T. D., Hilt, J. Z., Bhattacharyya, D., Hennig, B. (2014): Modulation of persistent organic pollutant toxicity through nutritional intervention: emerging opportunities in biomedicine and environmental remediation. - Science of the Total Environment 2014: 11-16.

[55] Petriello, M. C., Hoffman, J. B., Vsevolozhskaya, O., Morrisa, A. J., Hennigaf, B. (2018): Dioxin-like PCB 126 increases intestinal inflammation and disrupts gut microbiota and metabolic homeostasis. - Environmental Pollution 242: 1022-1032.

[56] Piskorska-Pliszczynska, J., Malagocki, P., Pajurek, M. (2019): Levels and trends of PCDD/Fs and DL-PCBs in Polish animal feeds, 2004-2017. - Food Additives \& Contaminants Part A: 1-17.

[57] Pocar, P., Fiandanese, N., Secchi, C., Berrini, A., Fischer, B., Schmidt, J. S., Schaedlich, K., Rhind, S. M., Zhang, Z., Borromeo, V. (2011): Effects of Polychlorinated Biphenyls in CD-1 mice: reproductive toxicity and intergenerational transmission. - Toxicological Sciences 126: 213-226.

[58] Rabinowitz, P., Conti, L. (2013): Links among human health, animal health, and ecosystem health. - Annual Review of Public Health 34: 189-204.

[59] Ray, P. D., Huang, B. W., Tsuji, Y. (2012): Reactive oxygen species (ROS) homeostasis and redox regulation in cellular signaling. - Cell Signal 24: 981-990.

[60] Round, J. L., Mazmanian, S. K. (2009): The gut microbiota shapes intestinal immune responses during health and disease. - Nature Reviews Immunology 9: 313-323.

[61] Saini, R. K., Keum, Y. S. (2018): Omega-3 and omega-6 polyunsaturated fatty acids: dietary sources, metabolism, and significance - a review. - Life Sciences 203: 255-267.

[62] Sapkota, A. R., Lefferts, L. Y., McKenzie, S., Walker, P. (2007): What do we feed to food-production animals? A review of animal feed ingredients and their potential impacts on human health. - Environmental Health Perspectives 115: 663-670.

[63] Sheng, Z. S., Wang, C., Ren, F., Liu, Y., Zhu, B. (2019): Molecular mechanism of endocrine-disruptive effects induced by Bisphenol A: the role of transmembrane Gprotein estrogen receptor 1 and integrin $\alpha \mathrm{v} \beta 3$. - Journal of Environmental Sciences 75: 113. 
[64] Shetty, S. A., Hugenholtz, F., Lahti, L., Smidt, H., de Vos, W. M. (2017): Intestinal microbiome landscaping: insight in community assemblage and implications for microbial modulation strategies. - FEMS Microbiology Review 41: 182-199.

[65] Smidt, H., de Vos, W. M. (2004): Anaerobic microbial dehalogenation. - Annual Review in Microbiology 58: 43-73.

[66] Spongberg, A. L., Witter, J. D. (2007): A review of PCB concentrations in tropical media, 1996-2007. - Revista de Biología Tropical 56: 1-9.

[67] Steinberg, R. M., Walker, D. M., Juenger, T. E., Woller, M. J., Gore, A. C. (2008): Effects of perinatal polychlorinated biphenyls on adult female rat reproduction: development, reproductive physiology, and second generational effects. - Biology of Reproduction 78: 1091-1101.

[68] Traag, V., Kan, K., Bovee, T., Weg, G. V. D., Onstenk, C., Portier L and Hoogenboom, R., Watanabe, M. X., Kunisue, T., Tao, L., Kannan, K., Subramanian, A., Tanabe, S., Iwata, H. (2010): Dioxin-like and perfluorinated compounds in pigs in an Indian open waste dumping site: toxicokinetics and effects on hepatic cytochrome P450 and blood plasma hormones. - Environmental Toxicology and Chemistry 29: 1551-1560.

[69] Weber, R., Herold, C., Hollert, H., Kamphues, J., Blepp, M., Ballschmiter, K. (2018): Reviewing the relevance of dioxin and PCB sources for food from animal origin and the need for their inventory, control and management. - Agricultural Sciences Europe 30: 42.

[70] Woodhouse, A. J., Cooke, G. M. (2004): Suppression of aromatase activity in vitro by PCBs 28 and 105 and Aroclor 1221. - Toxicology Letters 152: 91-100.

[71] Xu, W., Wang, X., Cai, Z. (2013): Analytical chemistry of the persistent organic pollutants identified in the Stockholm Convention: a review. - Analytica Chimica Acta 790: 1-13.

[72] Yim, Y. J., Seo, J., Kang, S. I., Ahn, J. H., Hur, H. G. (2008): Reductive dechlorination of methoxychlor and DDT by human intestinal bacterium Eubacterium limosum under anaerobic conditions. - Archives of Environmental Contamination and Toxicolology 54: 406-411.

[73] Yu, Y., Nie, F., Hay, A., Lin, H., Ma, Y., Ju, X., Gong, D., Chen, J. J., Gooneratne, R. (2017): Histopathological changes in zebrafish embryos exposed to DLPCBs extract from Zhanjiang coastal sediment. - Environmental Monitoring and Assessment 189: 289.

[74] Zhang, H. J., Jiang, X. J., Lu, L. P., Xiao, W. F. (2015a). Biodegradation of polychlorinated biphenyls (PCBs) by the novel identified Cyanobacterium anabaena PD1. - PLoS One 10: e0131450.

[75] Zhang, L., Nichols, R. G., Correll, J., Murray, I. A., Tanaka, N., Smith, P. B., Hubbard, T. D., Sebastian, A., Albert, I., Hatzakis, E., Gonzalez, F. J., Perdew, G., Patterson, A. D. (2015b). Persistent Organic Pollutants modify gut microbiota-host metabolic homeostasis in mice through aryl hydrocarbon receptor activation. - Environmental Health Perspectives 123: 679-688.

[76] Zijlstra, R. T., Beltranena, E. (2013): Swine convert co-products from food and biofuel industries into animal protein for food. - Animal Frontiers 3: 48-53.

[77] Zinsstag, J., Schelling, E., Waltnertoews, D., Tanner, M. (2011): From 'one medicine' to 'one health' and systemic approaches to health and well-being. - Preventive Veterinary Medicine 101: 148-156. 